$\operatorname{Nr} 60$

\author{
S. H. Saker, D. M. Abdou and I. KubiaczyK
}

\title{
OPIAL AND PÓLYA TYPE INEQUALITIES VIA CONVEXITY
}

\begin{abstract}
In this paper, we prove some new dynamic inequalities related to Opial and Pólya type inequalities on a time scale $\mathbb{T}$. We will derive the integral and discrete inequalities of Pólya's type as special cases and also derive several classical integral inequalities of Opial's type that has been obtained in the literature as special cases. The main results will be proved by using the chain rule, Hölder's inequality and Jensen's inequality, Taylor formula on time scales.
\end{abstract}

KEY WORDS: Opial's inequality, Hölder's inequality, Jensen's inequality, time scales.

AMS Mathematics Subject Classification: 26A15, 26A51, 26D10, 26D15, 39A13, 34A40.

\section{Introduction}

In 1960, Opial [12] proved the inequality

$$
\int_{0}^{h}\left|x(t) x^{\prime}(t)\right| d t \leq \frac{h}{4} \int_{0}^{h}\left|x^{\prime}(t)\right|^{2} d t
$$

where $x \in C^{1}[0, h], x(0)=x(h)=0$, and the constant $h / 4$ is the best possible. In 1960, Olech [11] extended the inequality (1) and proved that

$$
\int_{0}^{h}\left|x(t) x^{\prime}(t)\right| d t \leq \frac{h}{2} \int_{0}^{h}\left|x^{\prime}(t)\right|^{2} d t
$$

where $x(0)=0$ with equality if and only if $x(t)=$ ct. Since the discovery of these inequalities, they have been received non-diminishing attention and a large number of research papers giving their successively new proofs, providing various generalizations, and finding discrete analogues. For example, in 1965 Hua [8] generalized Opial's inequality (1) and proved that

$$
\int_{0}^{h}\left|x^{\prime}(t)\right|\left|x^{l}(t)\right| d t \leq \frac{h^{l}}{(l+1)} \int_{0}^{h}\left|x^{\prime}(t)\right|^{l+1} d t
$$


where $l$ is a positive integer. In 1966, Yang [20] proved a new generalization of Opial's inequality (1) of the form

$$
\int_{0}^{a}|x(t)|^{p}\left|x^{\prime}(t)\right|^{q} d t \leq \frac{q a^{p}}{p+q} \int_{0}^{a}\left|x^{\prime}(t)\right|^{p+q} d t, \text { where } p, q \geq 1 .
$$

Also in [20] Yang proved that if $x(\alpha)=0$, then

$$
\int_{\alpha}^{\beta} q(t)|x(t)|\left|x^{\prime}(t)\right| d t \leq \frac{1}{2} \int_{\alpha}^{\beta} \frac{1}{p(t)} d t \int_{\alpha}^{\beta} p(t) q(t)\left|x^{\prime}(t)\right|^{2} d t,
$$

where $p(t)$ is positive and continuos on $[\alpha, \beta]$ with $\int_{\alpha}^{\beta} \frac{1}{p(t)} d t<\infty$, and $q(t)$ is positive, bounded and non-decreasing on $[\alpha, \beta]$. In 1967, Maroni gave a new extension of Opial's inequality (1) of the form

(6) $\int_{\alpha}^{\beta}\left|x^{\prime}(t) x(t)\right| d t \leq \frac{1}{2}\left(\int_{\alpha}^{\beta} p^{1-\mu}(t) d t\right)^{2 / \mu}\left(\int_{\alpha}^{\beta} p(t)\left|x^{\prime}(t)\right|^{\nu} d t\right)^{2 / \nu}$,

where $\int_{\alpha}^{\beta} p^{1-\mu}(t) d t<\infty$ and $\mu>1$. In 1967, a sharper inequality was established by Godunova and Levin [7]. In particular, they proved that if $F$ is convex and an increasing function on $[0, \infty)$ with $F(0)=0$, then

$$
\int_{\alpha}^{\tau} F^{\prime}|x(t)|\left|x^{\prime}(t)\right| d t \leq F\left(\int_{\alpha}^{\tau}\left|x^{\prime}(t)\right| d t\right) .
$$

In 1972, Rozanova [14] obtained a new interesting generalization of (7). In particular, she proved that if $F, g$ are convex and nondecreasing functions on $(0, \infty)$, and $z(t) \geqslant 0, z^{\prime}(t)>0$ for $t \in[\alpha, \beta]$ such that $z(\alpha)=0$, then

$$
\begin{aligned}
\int_{\alpha}^{\beta} z^{\prime}(t) g\left(\frac{\left|x^{\prime}(t)\right|}{z^{\prime}(t)}\right) & F^{\prime}\left(z(t) g\left(\frac{|x(t)|}{z(t)}\right)\right) d t \\
\leq & F^{\prime}\left(\int_{\alpha}^{\beta} z^{\prime}(t) g\left(\frac{\left|x^{\prime}(t)\right|}{z^{\prime}(t)}\right) d t\right) .
\end{aligned}
$$

One of the extensions and generalizations of Opial's inequality (1) which involving $x^{(n)}(t), n \geqslant 1$ instead of $x^{\prime}(t)$ has been given by Willett [18]. In fact he proved that if $x(t) \in C^{n}[0, h]$ be such that $x^{(i)}(0)=0, i=0, \ldots, n-1$, then

$$
\int_{0}^{h}\left|x(t) x^{(n)}(t)\right| d t \leq \frac{h^{n}}{2} \int_{0}^{h}\left|x^{(n)}(t)\right|^{2} d t .
$$

In recent years the study of dynamic inequalities on time scales becomes a major field in pure and applied mathematics. The general idea is to prove 
an inequality where the domain of the unknown function is so-called time scale $\mathbb{T}$ which may be equal to the reals or to the integers. For more details on time scale analysis, we refer the reader to the two books by Bohner and Peterson $[5,6]$ which summarize and organize much of time scale calculus. In the following, for completness, we recall some related results to the inequalities that we will prove in this paper on time scales. In 2001, Bohner and Kaymakçalan initiated the study of dynamic inequalities of Opial-type on time scales and proved that if $x:[0, h]_{\mathbb{T}} \rightarrow \mathbb{R}$ is $\Delta$-differentiable with $x(0)=0$, then

$$
\int_{0}^{h}\left|x(t)+x^{\sigma}(t)\right|\left|x^{\Delta}(t)\right| \Delta t \leq h \int_{0}^{h}\left|x^{\Delta}(t)\right|^{2} \Delta t,
$$

where the time scale interval $[a, b]_{\mathbb{T}}$ is defined by $[a, b]_{\mathbb{T}}=\{t \in \mathbb{T}: a \leq t \leq b\}$. Since the discovery of the inequality (10), many papers which deal with new proofs, various generalizations, and extensions have appeared. For more details of Opial's type inequalities, we refer to the books $[2,3]$ and the papers $[15,16]$ and the references they are cited. In 2010, Karpuz [9] proved that

$$
\int_{\alpha}^{\tau} s(t)\left|\left(x^{2}(t)\right)^{\Delta}\right| \Delta t \leq K \int_{\alpha}^{\tau}\left|x^{\Delta}(t)\right|^{2} \Delta t
$$

where $s \in C_{r d}([\alpha, \tau],(0, \infty)), x \in C_{r d}^{1}\left([\alpha, \tau]_{\mathbb{T}}, \mathbb{R}\right)$ with $x(\alpha)=0$, and

$$
K=\sqrt{2 \int_{\alpha}^{\tau} s^{2}(t)(\sigma(t)-a) \Delta t} .
$$

Wong et al. [19] and Srivastava et al. [17] proved that if $s(t)$ is a positive and non-increasing function on $[a, b]_{\mathbb{T}}$, then

$$
\int_{a}^{b} s(t)|y(t)|^{\lambda}\left|x^{\Delta}(t)\right|^{\gamma} \Delta t \leq \frac{\gamma}{\lambda+\gamma}(b-a)^{\lambda} \int_{a}^{b} s(t)\left|x^{\Delta}(t)\right|^{\lambda+\gamma} \Delta t,
$$

where $x:[a, b]_{\mathbb{T}} \rightarrow \mathbb{R}$ is delta differentiable with $x(a)=0$.

Following this trend, in this paper, we will prove some new dynamic inequalities by using the properties of convex functions on time scales. The results as special cases, contain the results due to Rozanova, Godunova and Levin and Pólya when $\mathbb{T}=\mathbb{R}$. When $\mathbb{T}=\mathbb{N}$, we will derive some discrete inequalities which to the best of the authors knowledge has not be considered before. The paper is organized as follows: In Section 2, we present the definitions of time scales calculus that will be used in proving our main results. In Section 3, we will prove the main results. 


\section{Preliminaries}

The calculus on time scales was initiated by Aulbach and Hilger $[5,6]$ in order to create a theory that can unify discrete and continuos analysis. In this section, we present some concepts related to the notion of time scales. A time scale $\mathbb{T}$ is an arbitrary nonempty closed subset of the real numbers $\mathbb{R}$. We assume throughout that $\mathbb{T}$ has the topology that it inherits from the standard topology on the real numbers $\mathbb{R}$.

We define the forward jump operator $\sigma: \mathbb{T} \rightarrow \mathbb{T}$ by $\sigma(t):=\inf \{s \in$ $\mathbb{T}: s>t\}$, for $t \in \mathbb{T}$. The mapping $\mu: \mathbb{T} \rightarrow[0, \infty)$ defined by $\mu(t):=$ $\sigma(t)-t$ is called the graininess of $\mathbb{T}$. A point $t \in \mathbb{T}$ is said to be right-dense, right-scattered, if $\sigma(t)=t, \sigma(t)>t$, respectively. The backward jump operator $\rho: \mathbb{T} \rightarrow \mathbb{T}$ is defined by $: \rho(t):=\sup \{s \in \mathbb{T}: s<t\}$. A point $t \in \mathbb{T}$ is said to be left-dense, left-scattered, if $\rho(t)=t, \rho(t)<t$, respectively. A function $f: \mathbb{T} \rightarrow \mathbb{R}$ is called $r d$-continuous provided it is continuous at all right-dense points in $\mathbb{T}$ and its left-sided limits exist (finite) at all left-dense points in $\mathbb{T}$. The set of all such $r d$-continuous functions is denoted by $\mathrm{C}_{r d}(\mathbb{T})$. We define $f^{\sigma}:=f \circ \sigma$ and define the time scale interval $[a, b]_{\mathbb{T}}$ by $[a, b]_{\mathbb{T}}=\{t \in \mathbb{T}: a \leq t \leq b\}$.

Fix $t \in \mathbb{T}$ and let $f: \mathbb{T} \rightarrow \mathbb{R}$. Define $f^{\Delta}(t)$ to be the number (if it exists) with the property that given any $\epsilon>0$ there is a neighborhood $U$ of $t$ with

$$
\left|\left[f^{\sigma}(t)-f(s)\right]-f^{\Delta}(t)[\sigma(t)-s]\right| \leq \epsilon|\sigma(t)-s|, \text { for } s \in U \text {. }
$$

In this case, we say $f^{\Delta}(t)$ is the (delta) derivative of $f$ at $t$ and that $f$ is (delta) differentiable at $t$. We will make use of the following product and quotient rules for the derivative of the product $f g$ and the quotient $f / g$ (where $g g^{\sigma} \neq 0$, here $g^{\sigma}:=g \circ \sigma$ ) of two differentiable function $f$ and $g$

$$
(f g)^{\Delta}=f^{\Delta} g+f^{\sigma} g^{\Delta}=f g^{\Delta}+f^{\Delta} g^{\sigma}, \quad\left(\frac{f}{g}\right)^{\Delta}=\frac{f^{\Delta} g-f g^{\Delta}}{g g^{\sigma}} .
$$

A function $F: \mathbb{T} \rightarrow \mathbb{R}$ is an antiderivative of $f: \mathbb{T} \rightarrow \mathbb{R}$ if $F^{\Delta}(t)=f(t)$ for all $t \in \mathbb{T}$. In this case, we define the integral of $f$ by

$$
\int_{s}^{t} f(\tau) \Delta \tau=F(t)-F(s), \text { for } s, t \in \mathbb{T} .
$$

Now, we define the Taylor monomials or generalized polynomials as defined originally by Agarwal and Bohner [1]. The Taylor monomials $h_{k}: \mathbb{T} \times \mathbb{T} \rightarrow$ $\mathbb{R}, k \in \mathbb{N}_{0}=\mathbb{N} \cup\{0\}$, are defined recursively as follows: The function $h_{0}$ is defined by $h_{0}(t, s)=1$, for all $s, t \in \mathbb{T}$, and given $h_{k}$ for $k \in \mathbb{N}_{0}$, the function $h_{k+1}$ is defined by

$$
h_{k+1}(t, s)=\int_{s}^{t} h_{k}(\tau, s) \Delta \tau, \text { for all } s, t \in \mathbb{T} .
$$


If we let $h_{k}^{\Delta}(t, s)$ denote for each fixed $s \in \mathbb{T}$, the derivative of $h_{k}(t, s)$ with respect to $t$, then

$$
h_{k}^{\Delta}(t, s)=h_{k-1}(t, s), k \in \mathbb{N}, t \in \mathbb{T}, \text { for each fixed } s \in \mathbb{T} .
$$

The above definition obviously implies $h_{1}(t, s)=t-s$, for all $s, t \in \mathbb{T}$. For different formulae of $h_{k}(t, s)$, we refer the reader to [5]. We denote by $C_{r d}^{(n)}(\mathbb{T})$ the space of all functions $f \in C_{r d}(\mathbb{T})$ such that $f^{\Delta^{i}} \in C_{r d}(\mathbb{T})$ for $i=0,1,2, \ldots, n$ for $n \in \mathbb{N}$. For the function $f: \mathbb{T} \rightarrow \mathbb{R}$, we consider the second derivative $f^{\Delta^{2}}$ provided $f^{\Delta}$ is delta differentiable on $\mathbb{T}$ with derivative $f^{\Delta^{2}}=\left(f^{\Delta}\right)^{\Delta}$. Similarly, we define the $n^{t h}$ order derivative $f^{\Delta^{n}}=\left(f^{\Delta^{n-1}}\right)^{\Delta}$. The Taylor formula, see [1], which we will need to prove the main results in this paper, is given by

$$
f(t)=\sum_{k=0}^{n-1} f^{\Delta^{k}}(s) h_{k}(t, s)+\int_{s}^{t} h_{n-1}\left(t,(\sigma(\tau)) f^{\Delta^{n}}(\tau) \Delta \tau,\right.
$$

where $f \in C_{r d}^{(n)}(\mathbb{T})$ and $s \in \mathbb{T}$. The chain rule on a time scale $\mathbb{T}$, (see [5, Theorem 1.90]) states that: Let $f: \mathbb{R} \rightarrow \mathbb{R}$ be continuously differentiable and suppose that $g: \mathbb{T} \rightarrow \mathbb{R}$ is delta differentiable Then $f \circ g: \mathbb{T} \rightarrow \mathbb{R}$ is delta differentiable and the formula

$$
(f \circ g)^{\Delta}(t)=\int_{0}^{1} f^{\prime}\left[g(t)+h \mu(t) g^{\Delta}(t)\right] d h g^{\Delta}(t),
$$

holds. A special case of (14) is

$$
\left(f^{\gamma}(t)\right)^{\Delta}=\gamma f^{\Delta}(t) \int_{0}^{1}\left(h f^{\sigma}+(1-h) f\right)^{\gamma-1} d h .
$$

The Hölder's inequality on time scales [5, Theorem 6.13 ] is given by

$$
\int_{a}^{h}|f(t) g(t)| \Delta t \leq\left\{\int_{a}^{h}|f(t)|^{p} \Delta t\right\}^{\frac{1}{p}}\left\{\int_{a}^{h}|g(t)|^{q} \Delta t\right\}^{\frac{1}{q}} .
$$

where, $a, h \in \mathbb{T}, f, g \in C_{r d}(\mathbb{T}, \mathbb{R}), p>1$ and $q=p /(p-1)$. Jensen's inequality on time scales [6, Theorem 6.17$]$ is given by

$$
\phi\left(\frac{\int_{a}^{b} g(t) r(t) d t}{\int_{a}^{b} r(t) d t}\right) \leq \frac{\int_{a}^{b} \phi(g(t)) r(t) d t}{\int_{a}^{b} r(t) d t},
$$

where, $a, b \in \mathbb{T}, c, d \in \mathbb{R}, g, r \in C_{r d}\left([a, b]_{\mathbb{T}},(c, d)\right)$ and $\phi \in C((c, d), \mathbb{R})$ is a convex function. 


\section{Main results}

In this section, we will prove the main results . Throughout the paper, we will assume (sometimes without mentioning) that the functions are rd-continuous positive functions (and differentiable where appropriate) and the integrals are assumed to exist (and are finite). We begin with the following lemma which will be used in the proofs of our main results.

Lemma 1. Let $F$ be a convex and nonincreasing function and assume that $G$ is $\Delta$-differentiable function. If $G$ is increasing, then

$$
G^{\Delta}(x)\left(F^{\prime} \circ G\right)(x) \leq(F \circ G)^{\Delta}(x) .
$$

Proof. From the chain rule (14) for $h \in(0,1)$, we see that

$$
(F \circ G)^{\Delta}(x)=\left\{\int_{0}^{1} F^{\prime}\left[h G^{\sigma}(x)+(1-h) G(x)\right] d h\right\} G^{\Delta}(t)
$$

Since $G$ is an increasing function, $G(t) \leq G^{\sigma}(t)$ so for $h \in(0,1)$, we have

$$
\begin{aligned}
(F \circ G)^{\Delta}(x) & =\left\{\int_{0}^{1} F^{\prime}\left[h G^{\sigma}(x)+(1-h) G(x)\right] d h\right\} G^{\Delta}(t) \\
& \geq\left\{\int_{0}^{1} F^{\prime}[h G(x)+(1-h) G(x)] d h\right\} G^{\Delta}(t) \\
& =G^{\Delta}(x)\left(F^{\prime} \circ G\right)(x),
\end{aligned}
$$

which is the desired inequality. The proof is complete.

Theorem 1. Let $\mathbb{T}$ be a time scale with $\alpha, \beta \in \mathbb{T}$, and $z \in C_{r d}\left([\alpha, \beta]_{\mathbb{T}}, \mathbb{R}\right)$ is positive such that $z^{\Delta}(t)>0$. Suppose that $g, F$ be convex and increasing functions on $[0, \infty)$. If $x(t):[\alpha, \beta]_{\mathbb{T}} \rightarrow \mathbb{R}$ be $\Delta$-differentiable such that $x(\alpha)=0$, then

$$
\begin{array}{r}
\int_{\alpha}^{\beta} z^{\Delta}(t) g\left(\frac{\left|x^{\Delta}(t)\right|}{z^{\Delta}(t)}\right) F^{\prime}\left(z(t) g\left(\frac{|x(t)|}{z(t)}\right)\right) \Delta t \\
\leq F^{\prime}\left(\int_{\alpha}^{\beta} z^{\Delta}(t) g\left(\frac{\left|x^{\Delta}(t)\right|}{z^{\Delta}(t)}\right) \Delta t\right) .
\end{array}
$$

Proof. Consider $y(t)=\int_{\alpha}^{t}\left|x^{\Delta}(s)\right| \Delta s$. Then $y^{\Delta}(t)=\left|x^{\Delta}(t)\right|$ and $y(t) \geq|x(t)|$. Since $g$ is increasing, by using the Jensen inequality, we have that 


$$
\begin{aligned}
g\left(\frac{|x(t)|}{z(t)}\right) & \leq g\left(\frac{y(t)}{z(t)}\right) \leq g\left(\frac{\int_{\alpha}^{t} z^{\Delta}(s) \frac{\left|x^{\Delta}(t)\right|}{z^{\Delta}(t)} \Delta s}{\int_{\alpha}^{t} z^{\Delta}(s) \Delta s}\right) \\
& \leq \frac{1}{z(t)} \int_{\alpha}^{t} z^{\Delta}(s) g\left(\frac{y^{\Delta}(s)}{z^{\Delta}(s)}\right) \Delta s .
\end{aligned}
$$

Since $F$ is a convex function, we obtain

$$
\begin{aligned}
& \int_{\alpha}^{\beta} z^{\Delta}(t) g\left(\frac{\left|x^{\Delta}(t)\right|}{z^{\Delta}(t)}\right) F^{\prime}\left(z(t) g\left(\frac{|x(t)|}{z(t)}\right)\right) \Delta t \\
& \quad \leq \int_{\alpha}^{\beta} z^{\Delta}(t) g\left(\frac{y^{\Delta}(t)}{z^{\Delta}(t)}\right) F^{\prime}\left(\int_{\alpha}^{t} z^{\Delta}(s) g\left(\frac{y^{\Delta}(s)}{z^{\Delta}(s)}\right) \Delta s\right) \Delta t .
\end{aligned}
$$

By applying (18) on the right hand side of the last inequality, we get that

$$
\begin{aligned}
& \int_{\alpha}^{\beta} z^{\Delta}(t) g\left(\frac{\left|x^{\Delta}(t)\right|}{z^{\Delta}(t)}\right) F^{\prime}\left(z(t) g\left(\frac{|x(t)|}{z(t)}\right)\right) \Delta t \\
& \quad \leq \int_{\alpha}^{\beta}\left[F\left(\int_{\alpha}^{t} z^{\Delta}(s) g\left(\frac{y^{\Delta}(s)}{z^{\Delta}(s)}\right) \Delta s\right)\right]^{\Delta} \Delta t \\
& \quad=F\left(\int_{\alpha}^{\beta} z^{\Delta}(t) g\left(\frac{y^{\Delta}(t)}{z^{\Delta}(t)}\right) \Delta t\right)=F\left(\int_{\alpha}^{\beta} z^{\Delta}(t) g\left(\frac{\left|x^{\Delta}(t)\right|}{z^{\Delta}(t)}\right) \Delta t\right),
\end{aligned}
$$

which is the required inequality (19). The proof is complete.

Remark 1. We note that if the function $F$ is concave, then the reverse inequality in Theorem 1 also holds.

Remark 2. As a special case of Theorem 1 when $\mathbb{T}=\mathbb{R}$, we obtain Rozanova's inequality (8).

Remark 3. When $g(t)=t$, the inequality (19) reduces to the following inequality

$$
\int_{\alpha}^{\beta} F^{\prime}(|x(t)|)\left|x^{\Delta}(t)\right| \Delta t \leq F\left(\int_{\alpha}^{\beta}\left|x^{\Delta}(t)\right| \Delta t\right) .
$$

Remark 4. If we assume that $\mathbb{T}=\mathbb{R}$, and $g(t)=t$ in (19), then we obtain Godanova and Levin's inequality (7).

Remark 5. As a special case of Theorem 1 when $\mathbb{T}=\mathbb{R}, g(t)=t$, and $f(t)=t^{l+1}$, for $l \geq 0$, and $\alpha=0$ and $\beta=h$, we get Hua's inequality

$$
\int_{0}^{h}\left|x^{\prime}(t)\right|\left|x^{l}(t)\right| d t \leq \frac{h^{l}}{(l+1)} \int_{0}^{h}\left|x^{\prime}(t)\right|^{l+1} d t .
$$


Remark 6. As a special case of Theorem 1 when $\mathbb{T}=\mathbb{R}, g(t)=t$, we have that

$$
\int_{\alpha}^{\beta} F^{\prime}(|x(t)|)\left|x^{\prime}(t)\right| d t \leq F\left(\int_{\alpha}^{\beta}\left|x^{\prime}(t)\right| d t\right) .
$$

Remark 7. By using $F(u)=u^{2}$, and $x(t)=\int_{\alpha}^{t} \sqrt{q(s)}\left|y^{\prime}(s)\right| d s$, where $q(t)$ be positive, and non-increasing on $[\alpha, \beta]$, we get from (21) that

$$
2 \int_{\alpha}^{\beta}\left(\int_{\alpha}^{t} \sqrt{q(s)}\left|y^{\prime}(s)\right| d s\right) \sqrt{q(t)}\left|y^{\prime}(t)\right| d t \leq\left(\int_{\alpha}^{\beta} \sqrt{q(t)}\left|y^{\prime}(t)\right| d t\right)^{2} .
$$

Since

$$
\int_{\alpha}^{t} \sqrt{q(s)}\left|y^{\prime}(s)\right| d s \geqslant \sqrt{q(t)} \int_{\alpha}^{t}\left|y^{\prime}(s)\right| d s \geqslant \sqrt{q(t)} y(t),
$$

it follows that

$$
2 \int_{\alpha}^{\beta} q(t)|y(t)|\left|y^{\prime}(t)\right| d t \leq\left(\int_{\alpha}^{\beta} \frac{1}{\sqrt{p(t)}} \sqrt{p(t) q(t)}\left|y^{\prime}(t)\right| d t\right)^{2} .
$$

By applying the Cauchy-Schwarz inequality, we get that

$$
\int_{\alpha}^{\beta} q(t)|y(t)|\left|y^{\prime}(t)\right| d t \leq \frac{1}{2} \int_{\alpha}^{\beta} \frac{1}{p(t)} d t \int_{\alpha}^{\tau} p(t) q(t)\left|y^{\prime}(t)\right|^{2} d t,
$$

which is Yang's inequality (5).

Remark 8. For $\mathbb{T}=\mathbb{R}, g(t)=t$, and $F(u)=u^{2}$, the inequality (19) becomes

$$
\begin{aligned}
\int_{\alpha}^{\beta}\left|x^{\prime}(t) x(t)\right| d t & \leq \frac{1}{2}\left(\int_{\alpha}^{\beta}\left|x^{\prime}(t)\right| d t\right)^{2} \\
& =\frac{1}{2}\left(\int_{\alpha}^{\beta} p^{1 / \nu}(t) p^{-1 / \nu}(t)\left|x^{\prime}(t)\right| d t\right)^{2} .
\end{aligned}
$$

By applying Hölder's inequality with indices $\nu, \mu$ such that $\frac{1}{\nu}+\frac{1}{\mu}=1$, we have Maroni's inequality

$$
\int_{\alpha}^{\beta}\left|x^{\prime}(t) x(t)\right| d t \leq \frac{1}{2}\left(\int_{\alpha}^{\beta} p^{1-\mu}(t) d t\right)^{2 / \mu}\left(\int_{\alpha}^{\beta} p(t)\left|x^{\prime}(t)\right|^{\nu} d t\right)^{2 / \nu} .
$$

The proof of the following inequality follows immediately from the inequality (19) by applying Jensen's inequality. 
Theorem 2. Let $\mathbb{T}$ be a time scale with $\alpha, \beta \in \mathbb{T}$, and $z \in C_{r d}\left([\alpha, \beta]_{\mathbb{T}}, \mathbb{R}\right)$ is positive with $z^{\Delta}(t)>0$. Suppose that $F, g$ be convex and increasing functions on $[0, \infty)$. If $x(t):[\alpha, \beta]_{\mathbb{T}} \longrightarrow \mathbb{R}$ be $\Delta$-differentiable such that $x(\alpha)=0$, then

$$
\begin{aligned}
& \int_{\alpha}^{\beta} z^{\Delta}(t) g\left(\frac{\left|x^{\Delta}(t)\right|}{z^{\Delta}(t)}\right) F^{\prime}\left(z(t) g\left(\frac{|x(t)|}{z(t)}\right)\right) \Delta t \\
& \leq \frac{1}{\beta-\alpha} \int_{\alpha}^{\beta} F\left((\beta-\alpha) z^{\Delta}(t) g\left(\frac{\left|x^{\Delta}(t)\right|}{z^{\Delta}(t)}\right)\right) \Delta t .
\end{aligned}
$$

Theorem 3. Let $z \in C_{r d}\left([0, \tau]_{\mathbb{T}}, \mathbb{R}\right)$ is increasing with $z(0)=0$ and $\phi$ is convex and increasing function on $[0, \infty)$ such that $\phi(0)=0$. Suppose that $g, F$ be convex and increasing functions on $[0, \infty)$ and define $y(t)=$ $\int_{0}^{t} z^{\Delta}(s) g\left(\frac{\left|x^{\Delta}(s)\right|}{z^{\Delta}(s)}\right) \Delta s$ such that

$$
(F \circ y)^{\Delta}(t) \phi\left(\frac{1}{y^{\Delta}(t)}\right) \leq \frac{F(y(\tau))}{y(\tau)} \phi^{\prime}\left(\frac{t}{y(\tau)}\right) .
$$

If $x(t):[0, \tau]_{\mathbb{T}} \rightarrow \mathbb{R}$ be $\Delta$-differentiable such that $x(0)=0$ then

$$
\begin{aligned}
& \int_{0}^{\tau} F^{\prime}\left(z(t) g\left(\frac{|x(t)|}{z(t)}\right)\right) \psi\left(z^{\Delta}(t) g\left(\frac{\left|x^{\Delta}(t)\right|}{z^{\Delta}(t)}\right)\right) \Delta t \\
& \leq \Phi\left(\int_{0}^{\tau} z^{\Delta}(t) g\left(\frac{\left|x^{\Delta}(t)\right|}{z^{\Delta}(t)}\right) \Delta t\right),
\end{aligned}
$$

where $\psi(u)=u h(\phi(1 / u))$ and $\Phi(u)=F(u) h(\phi(\tau / u))$, where $h$ is a concave and increasing function on $[0, \infty)$.

Proof. Consider $y(t)=\int_{\alpha}^{t}\left|x^{\Delta}(s)\right| \Delta s$. Then $y^{\Delta}(t)=\left|x^{\Delta}(t)\right|$ and $y(t) \geq|x(t)|$. By applying Jensen's inequality, we have

$$
z(t) g\left(\frac{|x(t)|}{z(t)}\right) \leq z(t) g\left(\frac{y(t)}{z(t)}\right) \leq \int_{0}^{t} z^{\Delta}(s) g\left(\frac{\left|x^{\Delta}(s)\right|}{z^{\Delta}(s)}\right) \Delta s=y(t) .
$$

Then, we have

$$
\begin{aligned}
\int_{0}^{\tau} F^{\prime} & \left(z(t) g\left(\frac{|x(t)|}{z(t)}\right)\right) \psi\left(z^{\Delta}(t) g\left(\frac{\left|x^{\Delta}(t)\right|}{z^{\Delta}(t)}\right)\right) \Delta t \\
& \leq \int_{0}^{\tau} F^{\prime}(y(t)) \psi\left(y^{\Delta}(t)\right) \Delta t \\
& =\int_{0}^{\tau} F^{\prime}(y(t)) y^{\Delta}(t) h\left(\phi\left(\frac{1}{y^{\Delta}(t)}\right)\right) \Delta t .
\end{aligned}
$$


By applying (18), we see that

$$
\begin{aligned}
\int_{0}^{\tau} F^{\prime} & (y(t)) y^{\Delta}(t) h\left(\phi\left(\frac{1}{y^{\Delta}(t)}\right)\right) \Delta t \\
& \leq \frac{\int_{0}^{\tau}(F \circ y)^{\Delta}(t) h\left(\phi\left(\frac{1}{y^{\Delta}(t)}\right)\right) \Delta t}{\int_{0}^{\tau}(F \circ y)^{\Delta} \Delta t} \int_{0}^{\tau}(F \circ y)^{\Delta}(t) \Delta t \\
& \leq h\left(\frac{\int_{0}^{\tau}(F \circ y)^{\Delta}(t) \phi\left(\frac{1}{y^{\Delta}(t)}\right) \Delta t}{\int_{0}^{\tau}(F \circ y)^{\Delta} \Delta t}\right) F(y(\tau)) \\
& \leq h\left(\frac{\int_{0}^{\tau} \frac{F(y(\tau))}{y(\tau)} \phi^{\prime}\left(\frac{t}{y(\tau)}\right) \Delta t}{F(y(\tau))}\right) F(y(\tau))
\end{aligned}
$$

Since $\phi$ is convex, we can apply (19) and obtain

$$
\begin{aligned}
h\left(\frac{\int_{0}^{\tau} \frac{F(y(\tau))}{y(\tau)} \phi^{\prime}\left(\frac{t}{y(\tau)}\right) \Delta t}{F(y(\tau))}\right) F(y(\tau)) & =h\left(\phi\left(\frac{\tau}{y(\tau)}\right)\right) F(y(\tau)) \\
& =\Phi\left(\int_{0}^{\tau} z^{\Delta}(t) g\left(\frac{\left|x^{\Delta}(t)\right|}{z^{\Delta}(t)}\right) \Delta t\right) .
\end{aligned}
$$

This completes the proof.

As a special case of Theorem 3, if $g(t)=t, F(t)=\phi(t)=t^{2}$, and $h(t)=\sqrt{1+t}$, we get the following result.

Corollary 1. Assume that $x(t) \in C_{r d}\left([0, \tau]_{\mathbb{T}}, \mathbb{R}\right)$ be $\Delta$-differentiable such that $x(0)=0$. If $x^{\Delta}(t)>0$ and $x(\tau)=b$, then

$$
2 \int_{0}^{\tau} x(t)\left(1+\left(x^{\Delta}(t)\right)^{2}\right)^{1 / 2} \Delta t \leq b\left(b^{2}+\tau^{2}\right)^{1 / 2} .
$$

Remark 9. In Corollary 1 if $\mathbb{T}=\mathbb{R}$, then we obtain Pólya's inequality [13]

$$
2 \int_{0}^{\tau} x(t)\left(1+\left(x^{\prime}(t)\right)^{2}\right)^{1 / 2} d t \leq b\left(b^{2}+\tau^{2}\right)^{1 / 2} .
$$

Remark 10. In Corollary 1 if $\mathbb{T}=\mathbb{N}$, and $\tau=N+1$, then we obtain the discrete Pólya's inequality

$$
2 \sum_{n=0}^{N} x(n)\left(1+(\Delta x(n))^{2}\right)^{1 / 2} \leq b\left(b^{2}+(N+1)^{2}\right)^{1 / 2},
$$

such that $x(0)=0, \Delta x(n)>0$ and $x(N+1)=b$. 
In the following, we extend some of the above results to higher order dynamic inequalities.

Theorem 4. Let $\mathbb{T}$ be a time scale with $\alpha, \beta \in \mathbb{T}$. Suppose that $g, F$ be convex and increasing functions on $[0, \infty)$, and let $x(t) \in C_{r d}^{(n)}\left([\alpha, \beta]_{\mathbb{T}}, \mathbb{R}\right)$ be $\Delta$-differentiable such that $x^{(i)}(\alpha)=0$ for $i=0,1, \ldots, n-1$. Then

$$
\begin{aligned}
\int_{\alpha}^{\beta} z^{\Delta}(t) g\left(\frac{h_{n-1}(t, \alpha)\left|x^{\Delta^{n}}(t)\right|}{z^{\Delta}(t)}\right) F^{\prime}\left(z(t) g\left(\frac{|x(t)|}{z(t)}\right)\right) \Delta t \\
\leq \frac{1}{\beta-\alpha} \int_{\alpha}^{\beta} F\left((\beta-\alpha) z^{\Delta}(t) g\left(\frac{h_{n-1}(t, \alpha) y^{\Delta^{n}}(t)}{z^{\Delta}(t)}\right)\right) \Delta t .
\end{aligned}
$$

Proof. By setting,

$$
y(t)=\int_{\alpha}^{t} \int_{\alpha}^{t_{n-1}} \cdots \int_{\alpha}^{t_{1}}\left|x^{\Delta^{n}}(s)\right| \Delta s \Delta t_{1} \ldots \Delta t_{n-1},
$$

we see that

$$
y^{\Delta}(t), \ldots, y^{\Delta^{n}}(t) \geqslant 0, y^{\Delta^{n}}(t)=\left|x^{\Delta^{n}}(t)\right|, \text { and } y(t) \geqslant|x(t)| .
$$

From the Taylor's formula (13), and since $y^{\Delta^{i}}(\alpha)=0$, for $i=0,1, \ldots, n-1$, we have

$$
y(t) \leq \int_{\alpha}^{t} h_{n-1}(t, \sigma(s)) y^{\Delta^{n}}(s) \Delta s .
$$

Using the fact that $\left|h_{n}(t, s)\right|$ is nonincreasing with respect to its second component for $t \geqslant \sigma(s)>\alpha$, we have

$$
y(t) \leq h_{n-1}(t, \alpha) \int_{\alpha}^{t} y^{\Delta^{n}}(s) \Delta s .
$$

Since $g$ is convex, it follows from (29) that

$$
\begin{aligned}
g\left(\frac{|x(t)|}{z(t)}\right) & \leq g\left(\frac{y(t)}{z(t)}\right) \\
& \leq g\left(\frac{h_{n-1}(t, \alpha) \int_{\alpha}^{t} z^{\Delta}(s)\left(y^{\Delta^{n}}(s) / z^{\Delta}(s)\right) \Delta s}{\int_{\alpha}^{t} z^{\Delta}(s) \Delta s}\right) \\
& \leq \frac{1}{z(t)} \int_{\alpha}^{t} z^{\Delta}(s) g\left(\frac{h_{n-1}(t, \alpha) y^{\Delta n}(t)}{z^{\Delta}(s)}\right) \Delta s .
\end{aligned}
$$


From (29), we obtain

$$
\begin{gathered}
\int_{\alpha}^{\beta} z^{\Delta}(t) g\left(\frac{h_{n-1}(t, \alpha)\left|x^{\Delta^{n}}(t)\right|}{z^{\Delta}(t)}\right) F^{\prime}\left(z(t) g\left(\frac{|x(t)|}{z(t)}\right)\right) \Delta t \\
\int_{\alpha}^{\beta} z^{\Delta}(t) g\left(\frac{h_{n-1}(t, \alpha) y^{\Delta^{n}}(t)}{z^{\Delta}(t)}\right) F^{\prime}\left(z(t) g\left(\frac{y(t)}{z(t)}\right)\right) \Delta t \\
\leq \int_{\alpha}^{\beta} z^{\Delta}(t) g\left(\frac{h_{n-1}(t, \alpha) y^{\Delta^{n}}(t)}{z^{\Delta}(t)}\right) \\
\times F^{\prime}\left(\int_{\alpha}^{t} z^{\Delta}(s) g\left(\frac{h_{n-1}(t, \alpha) y^{\Delta^{n}}(s)}{z^{\Delta}(s)}\right) \Delta s\right) \Delta t .
\end{gathered}
$$

By applying (19), we have

$$
\begin{aligned}
\int_{\alpha}^{\beta} & z^{\Delta}(t) g\left(\frac{h_{n-1}(t, \alpha)\left|x^{\Delta^{n}}(t)\right|}{z^{\Delta}(t)}\right) F^{\prime}\left(z(t) g\left(\frac{|x(t)|}{z(t)}\right)\right) \Delta t \\
& \leq \int_{\alpha}^{\beta}\left[F \circ\left(\int_{\alpha}^{t} z^{\Delta}(s) g\left(\frac{h_{n-1}(t, \alpha) y^{\Delta^{n}}(s)}{z^{\Delta}(s)}\right) \Delta s\right)\right]^{\Delta} \Delta t \\
& =F \circ\left(\int_{\alpha}^{\beta} z^{\Delta}(t) g\left(\frac{h_{n-1}(t, \alpha) y^{\Delta^{n}}(t)}{z^{\Delta}(t)}\right) \Delta t\right) \\
& =F \circ\left(\int_{\alpha}^{\beta} z^{\Delta}(t) g\left(\frac{h_{n-1}(t, \alpha) x^{\Delta n}(t)}{z^{\Delta}(t)}\right) \Delta t\right) .
\end{aligned}
$$

By applying Jensen's inequality, we have

$$
\begin{aligned}
\int_{\alpha}^{\beta} & z^{\Delta}(t) g\left(\frac{h_{n-1}(t, \alpha)\left|x^{\Delta^{n}}(t)\right|}{z^{\Delta}(t)}\right) F^{\prime}\left(z(t) g\left(\frac{|x(t)|}{z(t)}\right)\right) \Delta t \\
& \leq F \circ\left(\frac{1}{\beta-\alpha} \int_{\alpha}^{\beta}(\beta-\alpha) z^{\Delta}(t) g\left(\frac{h_{n-1}(t, \alpha) y^{\Delta^{n}}(t)}{z^{\Delta}(t)}\right) \Delta t\right) \\
& \leq \frac{1}{\beta-\alpha} \int_{\alpha}^{\beta} F\left((\beta-\alpha) z^{\Delta}(t) g\left(\frac{h_{n-1}(t, \alpha) y^{\Delta^{n}}(t)}{z^{\Delta}(t)}\right)\right) \Delta t
\end{aligned}
$$

which is the desired inequality. The proof is complete.

Remark 11. As a special case of Theorem 4 when $\mathbb{T}=\mathbb{R}, g(t)=$ $t, z(t)=1$, and $F(u)=u^{2}$, we obtain

$$
\int_{\alpha}^{\beta}|x(t)|\left|x^{(n)}(t)\right| d t \leq \frac{(\beta-\alpha)^{n}}{2(n-1) !} \int_{\alpha}^{\beta}\left|x^{(n)}(t)\right|^{2} d t .
$$

which improves Willett's inequality (9). 
Theorem 5. Let $\mathbb{T}$ be a time scale with $\alpha, \beta \in \mathbb{T}$. Suppose that $F$ be a convex and an increasing function on $[0, \infty)$, let $x(t) \in C_{r d}^{(n)}\left([\alpha, \beta]_{\mathbb{T}}, \mathbb{R}\right)$ be $\Delta$-differentiable, satisfying $x(\alpha)=0$, then we have the inequality

$$
\begin{aligned}
& \int_{\alpha}^{\beta}|y(t)|^{1-p} F^{\prime}(|y(t)|)\left|y^{\Delta^{n}}(t)\right|^{p} \Delta t \\
& \quad \leq \frac{p}{C^{q}} F\left(C\left(\int_{\alpha}^{\beta}\left|y^{\Delta^{n}}(t)\right|^{p} \Delta t\right)^{1 / p}\right),
\end{aligned}
$$

where $\left(\int_{\alpha}^{t}\left(h_{n-1}(t, \sigma(s))\right)^{q}\right)^{1 / q} \leq C$ and $1 / p+1 / q=1$ for $p>1$.

Proof. Proceeding as in the proof of Theorem 4 by using Taylor's formula (13), and since $y^{\Delta^{i}}(\alpha)=0$, for $i=0,1, \ldots, n-1$, we have $y(t) \leq$ $\int_{\alpha}^{t} h_{n-1}(t, \sigma(s)) y^{\Delta^{n}}(s) \Delta s$. Applying Holder's inequality with indices $p, q$ in the right side of the above inequality, we obtain

$$
\begin{aligned}
y(t) & \leq\left(\int_{\alpha}^{t}\left(h_{n-1}(t, \sigma(s))\right)^{q}\right)^{1 / q}\left(\int_{\alpha}^{t}\left|y^{\Delta^{n}}(s)\right|^{p} \Delta s\right)^{1 / p} \\
& \leq C\left(\int_{\alpha}^{t}\left|y^{\Delta^{n}}(s)\right|^{p} \Delta s\right)^{1 / p},
\end{aligned}
$$

Let $z(t)=\int_{\alpha}^{t}\left|y^{\Delta^{n}}(s)\right|^{p} \Delta s$. Then $z(\alpha)=0$ and $\left|y^{\Delta^{n}}(t)\right|^{p}=z^{\Delta}(t)$. From this and (31), we have

$$
\begin{aligned}
& \int_{\alpha}^{\beta}|y(t)|^{1-p} F^{\prime}(|y(t)|)\left|y^{\Delta^{n}}(t)\right|^{p} \Delta t \\
& \quad \leq \int_{\alpha}^{\beta} C^{1-p}(z(t))^{-1 / q} F^{\prime}\left(C(z(t))^{1 / p}\right) z^{\Delta}(t) \Delta t .
\end{aligned}
$$

By applying the chain rule (17) we see (note that $z(t)>0$ and $z^{\Delta}(t)>0$ ), that

$$
\begin{aligned}
\left(z^{1 / p}(t)\right)^{\Delta} & =\frac{1}{p} z^{\Delta}(t) \int_{0}^{1}\left(h z^{\sigma}+(1-h) z\right)^{-1 / q} d h \\
& \geq \frac{1}{p} z^{\Delta}(t)(z(t))^{-1 / q} .
\end{aligned}
$$

Substituting (33) into (32), we have

$$
\begin{aligned}
& \int_{\alpha}^{\beta}|y(t)|^{1-p} F^{\prime}(|y(t)|)\left|y^{\Delta^{n}}(t)\right|^{p} \Delta t \\
& \quad \leq \frac{p}{C^{p}} \int_{\alpha}^{\beta} F^{\prime}\left(C(z(t))^{1 / p}\right)\left(C\left(z^{1 / p}(t)\right)\right)^{\Delta} \Delta t .
\end{aligned}
$$


By applying (19) on the last inequality, we have

$$
\begin{array}{r}
\int_{\alpha}^{\beta}|y(t)|^{1-p} F^{\prime}(|y(t)|)\left|y^{\Delta^{n}}(t)\right|^{p} \Delta \leq \frac{p}{C^{q}} F\left(\int_{\alpha}^{\beta}\left(C z^{1 / p}(t)\right)^{\Delta} \Delta t\right) \\
=\frac{p}{C^{q}} F\left(C\left(z^{1 / p}(\beta)\right)\right)=\frac{p}{C^{q}} F\left(C\left(\int_{\alpha}^{\beta}\left|y^{\Delta^{n}}(t)\right|^{p} \Delta t\right)^{1 / p}\right),
\end{array}
$$

which is the desired inequality. The proof is complete.

\section{References}

[1] Agarwal R.P., Bohner M., Basic Calculus on Time Scales and Some of its Applications, Results Math., 35(1999), 3-22.

[2] Agarwal R.P., Pang P.Y.H., Opial Inequalities with Applications in Differential and Difference Equations, Kluwer, Dordrechet 1995.

[3] Agarwal R.P., O'Regan D., Saker S.H., Dynamic Inequalities on Time Scales, Springer, Switzerland, 2014.

[4] Bohner M., Kaymakçalan B., Opial inequalities on time scales, Ann. Polon. Math., 77(2001), 11-20.

[5] Bohner M., Peterson A., Dynamic Equations on Time Scales: An introduction With Applications, Birkhäuser, Boston, 2001.

[6] Bohner M., Peterson A., Advances in Dynamic Equations on Time Scales, Birkhäuser, Boston, 2003.

[7] Godunova E.K., Levin V.I., On an inequality of Maroni, Mat. Zametki, 2 (1967), 221-224.

[8] Hua L.K., On an inequality of Opial, Sci China., 14(1965), 789-790.

[9] Karpuz B., Kaymakçalan B., ÖCalan Ö., A generalization of Opial's inequality and applications to second-order dynamic equations, Diff. Eqns. Dyn. Sys., 18(2010), 11-18.

[10] Maroni P., Sur I'inégalité d'Opial-Beesack, C. R. Acad. Sci. Paris Sér., 264 (1967), A62-A64.

[11] Olech Z., A simple proof of a certain result of Z. Opial, Ann. Polon. Math., 8(1960), 61-63.

[12] Opial Z., Sur une inégalité, Ann. Polon. Math., 8(1960), 29-32.

[13] Pólya P., Problem 4264, Amer. Math. Monthly, 54(1947), 479.

[14] Rozanova G.I., Integral inequalities with derivatives and with arbitrary convex functions, Mos. Gos. Ped. Inst. Vcen. Zap., 460(1972), 58-65.

[15] Saker S.H., Some Opial-type inequalities on time scales, Abstr. Appl. Anal., Art. no. 265316, (2011), 19 pages.

[16] SAKER S.H., New inequalities of Opial's type on time scales and some of their applications, Discrete Dynamics in Nature and Society, vol. 2012, Article ID 362526,(2012), 23 pages.

[17] Srivastava H.M., Tseng K.L., Tseng S.J., Lo J.C., Some weighted Opial-type inequalities on time scale, Taiwanese Journal of Mathematics, 14(1)2010), 107-122. 
[18] Willett D., The existence-uniqueness theorem for an nth order linear ordinary differential equation, Amer. Math. Monthly, 75(2)(1968), 174-178.

[19] Wong F.H., Lin W.C., Yu S.L., YeH , Some generalizations of Opial's inequalities on time scales, Taiwanese Journal of Mathematics, 12(2)(2008), 463-471.

[20] Yang G.S., On a certain result of Z. Opial, Proc. Japan Acad., 42(1966), 78-83.

\author{
S. H. SAKER \\ Department of MATHEMATics \\ FACULTY OF SCIENCE \\ MANSOURA UNIVERSITY \\ MANSOURA 35516, EGYPT \\ e-mail: shsaker@mans.edu.eg \\ D. M. ABdou \\ Department of Mathematics \\ FaCUlty of SCIEnCE \\ Al-Azhar University \\ NAsR City 11884, EgYPT \\ e-mail: abdoudoaa@yahoo.com \\ I. KUBIACZYK \\ Faculty of Mathematics and Computer Science \\ ADAM MiCKIEWICZ UNIVERSITY \\ Umultowska 87, 61-614 Poznan, Poland \\ e-mail: kuba@amu.edu.pl
}

Received on 28.01.2018 and, in revised form, on 15.06.2018. 